\title{
Mixed Active/Passive Robust Fault Detection and Isolation Using Set-Theoretic Unknown Input Observers
}

\author{
Feng $\mathrm{Xu}^{\circledR}$, Junbo Tan, Xueqian Wang, Vicenç Puig ${ }^{\circledR}$, Bin Liang, and Bo Yuan, Member, IEEE
}

\begin{abstract}
This paper proposes a robust fault detection and isolation (FDI) approach that combines active and passive robust FDI approaches. Standard active FDI approaches obtain robustness by using the unknown input observer (UIO) to decouple unknown inputs from residuals. Differently, standard passive FDI approaches achieve robustness by using the set theory to bound the effect of uncertain factors (disturbances and noises). In this paper, we combine the UIO-based and the set-based approaches to produce a mixed robust FDI, which can mitigate the disadvantages and exert the advantages of the two robust FDI approaches. In order to emphasize the role of set theory, the UIO design based on the set theory is named as the set-theoretic UIO (SUIO). A quadrotor subsystem is used to illustrate the effectiveness of the proposed FDI approach.
\end{abstract}

Note to Practitioners-The proposed SUIO-based approach achieves robustness in FD against disturbances, noises, and modeling uncertainties (e.g., linearization errors) either by decoupling or by bounding their effect on the residuals. Moreover, this approach allows FI by generating a set of structured residuals decoupling the effect of faults. The authors think that this method can be used for processes that operate around equilibrium points. In this case, we can linearize the nonlinear systems into linear systems with bounded unknown inputs and then apply the proposed method. Moreover, an important feature of this method is that it can overcome the design condition of the standard UIO approach, which means that it could be applied to a larger number of applications.

Index Terms-Active and passive decoupling, robust fault detection and isolation (FDI), set theory, unknown input observer (UIO).

Manuscript received June 21, 2017; revised November 11, 2017; accepted November 16, 2017. Date of publication December 20, 2017; date of current version April 5, 2018. This paper was recommended for publication by Associate Editor C. N. Hadjicostis and Editor S. Reveliotis upon evaluation of the reviewers' comments. This work was supported in part by the National Natural Science Foundation of China under Grant 61673239, in part by the Natural Science Foundation of Guangdong Province under Grant 2014A030310318 and Grant 2015A030313881, in part by the Basic Research of Shenzhen under Grant JCYJ20170412171459177, Grant JCYJ20160301100921349, Grant JSGG20160301100206969, and Grant JSGG20150331151358134, and in part by the Spanish Government (Ministerio de Economia y Competitividad) and FEDER under Project DPI201458104-R (HARCRICS). (Corresponding authors: Xueqian Wang; Bin Liang.)

F. Xu, J. Tan, and X. Wang are with the Center of Intelligent Control and Telescience, Graduate School at Shenzhen, Tsinghua University, Shenzhen 518055, China (e-mail: xu.feng@sz.tsinghua.edu.cn; tjb16@mails.tsinghua.edu.cn; wang.xq@sz.tsinghua.edu.cn).

V. Puig is with the Institut de Robòtica i Informàtica Industrial, Technical University of Catalonia, Llorens i Artigas, 08028 Barcelona, Spain (e-mail: vicenc.puig@upc.edu).

B. Liang is with the Tsinghua National Laboratory for Information Science and Technology, Tsinghua University, Beijing 100084, China (e-mail: bliang@tsinghua.edu.cn).

B. Yuan is with the Intelligent Computing Laboratory, Division of Informatics, Graduate School at Shenzhen, Tsinghua University, Shenzhen 518055, China (e-mail: yuanb@sz.tsinghua.edu.cn).

Color versions of one or more of the figures in this paper are available online at http://ieeexplore.iee.org.

\section{INTRODUCTION}

A S THE complexity of technical systems increases, diagnosis of faults occurred in the systems (e.g., aircrafts, manipulators, etc.) has been attracting more and more attention in order to improve dependability and reliability [1], [2]. However, since real systems are affected by uncertain factors (disturbances, noises, modeling errors, etc.), an effective fault detection and isolation (FDI) approach must be able to deal with them. An FDI method is called robust if it can separate the effects of faults and uncertain factors on the residuals from each other.

Robust FDI is classified into passive and active methods [1], [3]-[6]. In the active robust FDI approaches, a well-known technique is based on the unknown input observer (UIO) [3], [7]-[9]. Since the UIO can be designed to be insensitive to unknown inputs, it can be used to generate residual signals that are only sensitive to faults, but insensitive to the unknown inputs (uncertain factors). In this way, FDI robustness with respect to the unknown inputs can be achieved. However, a complete decoupling of all unknown inputs must satisfy the design condition of UIO [3], [10], which can only be ensured by a limited number of practical systems. Thus, in order to overcome this restriction, different methods were further proposed based on different assumptions on the knowledge of unknown inputs (see [11]-[13]).

In the passive robust FDI methods (mainly the set-based approaches), the effects of uncertain factors are propagated to the residuals by considering theirs bounds. There exist three types of set-based approaches, which use set invariance theory [14]-[16], interval observers [17], [18], and set-membership estimation [19]-[21], respectively. By propagating the effects of uncertain factors into the residuals, they generate fixed (invariant set-based approaches) or adaptive (interval observerbased approaches and set-membership estimation approaches) thresholds for the residuals. The main advantage of the setbased approaches consists in achieving robust FDI requiring only the boundedness assumption of uncertain factors. Thus, if the set-based approaches have detected an inconsistency between the model and the measurements, it implies that the system has become faulty. However, FDI decisions of the set-based approaches are more conservative than the active robust FDI approaches due to the consideration of the worst case situation of uncertain factors, being their main weakness.

Considering the advantages and weaknesses of the active and passive FDI methods, this paper aims to propose a mixed active/passive robust FDI method in order to mitigate their disadvantages and exert their advantages with the aim of obtaining two important benefits as follows.

1) With respect to the set-based passive FDI methods, the proposed approach can reduce FDI conservatism 
because the effect of some unknown inputs that could not be bounded can be actively decoupled. This results in tighter residual bounds and smaller detectable faults;

2) With respect to the UIO-based active FDI methods, the proposed approach can extend the applicability to those systems that cannot satisfy the standard UIO design condition. This is achieved by dividing unknown inputs into two groups: one group containing unknown inputs that can be actively decoupled and the other group whose effect is bounded instead of being decoupled.

Furthermore, assuming that there are $p$ actuators installed in the system, the proposed approach in this paper uses $p$ theoretic UIOs (SUIOs) to achieve not only FD but also FI, designing each UIO to be insensitive to one known input and several unknown inputs. This paper is based on preliminary results in [22] and has the following three important features with respect to the existing FDI schemes.

1) Since each SUIO is designed to be insensitive to faults in a particular actuator but sensitive to faults in the remaining $p-1$ actuators, this means that using the proposed FDI scheme, faults in any actuator can be monitored by $p-1$ SUIOs together, which has higher fault sensitivity than some traditional observer-based FDI schemes only relying on one observer to monitor each actuator;

2) When designing an SUIO to be insensitive to one input generated by an actuator, whether the actuator is healthy or faulty cannot affect the residual of this SUIO. Thus, each SUIO can simultaneously match the healthy system situation and one faulty situation of the corresponding actuator. It is not needed to design an extra observer to especially monitor the healthy situation as in the traditional observer-based FDI schemes. Compared with some traditional schemes with a bank of observers (interval observer, set-membership estimator, etc.), the proposed FDI scheme needs one observer less (i.e., only $p$ observers);

3) With respect to the UIO-based FI methods, the proposed method establishes guaranteed FI conditions using invariant sets. For the considered faults, we can check the guaranteed FI conditions to know whether they are isolable in advance and offline. This is a feature that the standard UIO-based approach cannot provide.

The remainder of this paper is organized as follows. Section II introduces the plant model and UIO. Section III introduces the method designing SUIOs, based on a mixed active/passive decoupling method. Section IV illustrates the effectiveness of the proposed method using a quadrotor subsystem. This paper is concluded in Section $\mathrm{V}$ including directions for the future work.

\section{SySTEM DESCRIPTION}

\section{A. Model of Plant}

The linear discrete time-invariant plant under the effect of actuator faults is modeled as

$$
\begin{aligned}
x_{k+1} & =A x_{k}+b_{1} f_{1} u_{k}^{1}+\cdots+b_{p} f_{p} u_{k}^{p}+E \omega_{k} \\
y_{k} & =C x_{k}+F \eta_{k}
\end{aligned}
$$

where $A \in \mathbb{R}^{n \times n}, b_{i} \in \mathbb{R}^{n}$ is the $i$ th column of the input matrix $B=\left[b_{1}, \ldots b_{i}, \ldots, b_{p}\right] \in \mathbb{R}^{n \times p}, E \in \mathbb{R}^{n \times r}, C \in$ $\mathbb{R}^{q \times n}$, and $F \in \mathbb{R}^{q \times s}$ are time-invariant matrices, $k$ denotes the $k$ th discrete-time instant, $x_{k} \in \mathbb{R}^{n}$ and $y_{k} \in \mathbb{R}^{q}$ are the state and output vectors, $u_{k}=\left[u_{k}^{1}, \ldots, u_{k}^{i}, \ldots, u_{k}^{p}\right]^{T} \in \mathbb{R}^{p}\left(u_{k}^{i}\right.$ is the $i$ th component of $u_{k}$ associated with the $i$ th actuator) and $\omega_{k} \in \mathbb{R}^{r}$ are known and unknown inputs (process disturbances, modeling errors, etc.), $\eta_{k} \in \mathbb{R}^{s}$ represents the noise vector, and $f_{i}$ models the fault magnitude in the $i$ th actuator.

When the $i$ th actuator is healthy, $f_{i}$ takes the value 1 , while if the $i$ th actuator becomes faulty, $f_{i}$ takes a value inside the interval $[0,1)$, where 0 means that the $i$ th actuator has completely lost its function and a value inside $(0,1)$ means that the $i$ th actuator has partially lost its performance.

Assumption 1: There is only one actuator that may become faulty at a time and faults are persistent such that the FDI module has sufficient time to detect and isolate it.

Assumption 2: The measurement noise vector $\eta_{k}$ is bounded by a known set $V=\left\{\eta \in \mathbb{R}^{s}:\left|\eta-\eta^{c}\right| \leq \bar{\eta}\right\}$, where $\eta^{c}$ and $\bar{\eta}$ are known and constant vectors.

When the $i$ th actuator becomes faulty while all the other actuators are still healthy, the model (1) can be rewritten as

$$
\begin{aligned}
x_{k+1} & =A x_{k}+B_{i} \mathcal{F}_{i} u_{k}+b_{i} f_{i} u_{k}^{i}+E \omega_{k} \\
y_{k} & =C x_{k}+F \eta_{k}
\end{aligned}
$$

where $B_{i}=\left[b_{1}, \ldots, b_{i-1}, 0, b_{i+1}, \ldots, b_{p}\right] \in \mathbb{R}^{n \times p}$ and $\mathcal{F}_{i}=\operatorname{diag}(1, \ldots, 1,0,1, \ldots, 1) \in \mathbb{R}^{p \times p}$, where 0 is the $i$ th diagonal element of $\mathcal{F}_{i}$. Since the actuator faults are treated as unknown inputs, (2a) can be further rewritten as

$$
x_{k+1}=A x_{k}+\mathcal{B}_{i} u_{k}+\mathcal{E}_{i} \omega_{k}^{i}
$$

where $\mathcal{B}_{i}=B_{i} \mathcal{F}_{i}, \mathcal{E}_{i}=\left[\begin{array}{ll}b_{i} & E\end{array}\right]$ and $\omega_{k}^{i}=\left[\begin{array}{ll}f_{i} u_{k}^{i} & \omega_{k}^{T}\end{array}\right]^{T}$.

Remark 3: The model (2) or (3) can represent the healthy system situation as well when $f_{i}$ takes the value 1 .

\section{B. Notion of UIOs}

When the plant is in the healthy situation, (2) can be equivalently rewritten as

$$
\begin{aligned}
x_{k+1} & =A x_{k}+B u_{k}+E \omega_{k} \\
y_{k} & =C x_{k}+F \eta_{k} .
\end{aligned}
$$

Based on (4), the UIO matching the healthy situation of the plant can be designed as

$$
\begin{aligned}
z_{k+1} & =N z_{k}+T u_{k}+K y_{k} \\
\hat{x}_{k} & =M z_{k}+H y_{k} \\
\hat{y}_{k} & =C \hat{x}_{k}
\end{aligned}
$$

where $z_{k} \in \mathbb{R}^{n}, \hat{x}_{k} \in \mathbb{R}^{n}$, and $\hat{y}_{k} \in \mathbb{R}^{q}$ are the state vector of the UIO, the state, and output estimation vectors of the plant, respectively, and $N \in \mathbb{R}^{n \times n}, T \in \mathbb{R}^{n \times p}, K \in \mathbb{R}^{n \times q}$, $M \in \mathbb{R}^{n \times n}$, and $H \in \mathbb{R}^{n \times q}$ are parametric matrices.

With (4) and (5), the corresponding state estimation error vector is defined as

$$
e_{k}=x_{k}-\hat{x}_{k}
$$


with the dynamics described by

$$
\begin{aligned}
e_{k+1}= & \left(A-H C A-M K_{1} C\right) e_{k} \\
& +\left[\left(A-H C A-M K_{1} C\right) M-M N\right] z_{k} \\
& +\left[\left(A-H C A-M K_{1} C\right) H-M K_{2}\right] y_{k} \\
& +(B-M T-H C B) u_{k}+(E-H C E) w_{k} \\
& -H F \eta_{k+1}-M K_{1} F \eta_{k}
\end{aligned}
$$

where $K=K_{1}+K_{2}$. From (7), the parametric matrices of the UIO can be obtained by solving

$$
\begin{aligned}
E-H C E & =\mathbf{0} \\
B-M T-H C B & =\mathbf{0} \\
\left(A-H C A-M K_{1} C\right) M-M N & =\mathbf{0} \\
\left(A-H C A-M K_{1} C\right) H-M K_{2} & =\mathbf{0} .
\end{aligned}
$$

Then, under (8), the dynamics of $e_{k}$ can be reduced into

$$
e_{k+1}=\left(A-H C A-M K_{1} C\right) e_{k}-H F \eta_{k+1}-M K_{1} F \eta_{k} .
$$

Note that the satisfaction of (8) implies that the UIO is not sensitive to $\omega_{k}$. However, it can also be observed in (9) that the UIO is still sensitive to the measurement noises, because the satisfaction of (8) does not imply the satisfaction of $H F=\mathbf{0}$ and $M K_{1} F=\mathbf{0}$. This is a known issue in the available UIO literature. In this paper, instead of actively decoupling the noises, they will be bounded using the set theory. By analyzing (7) and (8), the conditions to ensure the existence of the UIO (5) [i.e., the satisfaction of (8)] are given in Theorem 4.

Theorem 4 (see [3]): Necessary and sufficient existence conditions of the UIO (5) for the system (2) are: 1) $\operatorname{rank}(C E)=\operatorname{rank}(E)$ and 2$)\left(C, A_{1}\right)$ is a detectable pair, where $A_{1}=A-E\left[(C E)^{T} C E\right]^{-1}(C E)^{T} C A$.

\section{Robust FAult Detection AND IsOlation}

\section{A. Design of SUIOs}

According to Theorem 4, if a system cannot satisfy the existence condition of UIOs, it is impossible to design conventional UIOs (as in [3]) to decouple all unknown inputs and implement robust FDI, which restricts the applicability of the UIO-based method. In order to avoid this limitation, we propose to divide the unknown input vector $\omega_{k}^{i}$ into two groups: $\omega_{k}^{i, 1}$ and $\omega_{k}^{i, 2}$, where $\omega_{k}^{i, 1}$ represents a vector composed of unknown inputs that can be actively decoupled by a UIO, while $\omega_{k}^{i, 2}$ includes those that cannot be actively decoupled.

Remark 5: Since the actuator faults are treated as unknown inputs in this paper, for faults in the $i$ th actuator, the partitioning of $\omega_{k}^{i}$ into $\omega_{k}^{i, 1}$ and $\omega_{k}^{i, 2}$ must be done to guarantee that $\omega_{k}^{i, 1}$ includes $f_{i} u_{k}^{i}$ as one of its components and there exist UIOs that can actively decouple $\omega_{k}^{i, 1}$.

Considering that the dimension of $\omega_{k}^{i, 1}$ is $n_{a}^{i}$, where $n_{a}^{i}$ denotes the number of unknown inputs that can be actively decoupled by a UIO, $n_{p}^{i}=r+1-n_{a}^{i}$ represents the dimension of $\omega_{k}^{i, 2}$, which is the number of the remaining unknown inputs that the UIO cannot be insensitive to. Furthermore, the matrix $\mathcal{E}_{i}$ corresponding to $\omega_{k}^{i}$ can be divided into $\mathcal{E}_{i}=\left[\begin{array}{ll}\mathcal{E}_{i}^{1} & \mathcal{E}_{i}^{2}\end{array}\right]$, where $\mathcal{E}_{i}^{1} \in \mathbb{R}^{n \times n_{a}^{i}}$ and $\mathcal{E}_{i}^{2} \in \mathbb{R}^{n \times n_{p}^{i}}$. Since $n_{a}^{i}$ denotes the number of unknown inputs that a UIO can be designed to be insensitive to, we can design a UIO able to decouple $\omega_{k}^{i, 1}$ instead of $\omega_{k}^{i}$, which means that the conditions in Theorem 4 can be overcome due to the partial decoupling unknown inputs.

The UIO for the $i$ th actuator can be denoted as

$$
\begin{aligned}
z_{k+1}^{i} & =N^{i} z_{k}^{i}+T^{i} u_{k}+K^{i} y_{k} \\
\hat{x}_{k}^{i} & =M^{i} z_{k}^{i}+H^{i} y_{k} \\
\hat{y}_{k}^{i} & =C \hat{x}_{k}^{i}
\end{aligned}
$$

where $z_{k}^{i}, \hat{x}_{k}^{i}$, and $\hat{y}_{k}^{i}$ have similar definitions as in (5), and $N^{i}$, $T^{i}, K^{i}, M^{i}$, and $H^{i}$ denote the corresponding UIO parametric matrices. The UIO (10) is designed to be insensitive to the unknown input vector $\omega_{k}^{i, 1}$, but sensitive to $\omega_{k}^{i, 2}$.

By considering (3) and (7), we can obtain that the state estimation error of the $i$ th UIO (10) as

$$
\begin{aligned}
e_{k+1}^{i}= & \left(A-H^{i} C A-M^{i} K_{1}^{i} C\right) e_{k}^{i} \\
& +\left[\left(A-H^{i} C A-M^{i} K_{1}^{i} C\right) M^{i}-M^{i} N^{i}\right] z_{k} \\
& +\left[\left(A-H^{i} C A-M^{i} K_{1}^{i} C\right) H-M^{i} K_{2}^{i}\right] y_{k} \\
& +\left(\mathcal{B}_{i}-M^{i} T^{i}-H^{i} C \mathcal{B}_{i}\right) u_{k} \\
& +\left(\mathcal{E}_{i}^{1}-H^{i} C \mathcal{E}_{i}^{1}\right) \omega_{k}^{i, 1} \\
& +\left(\mathcal{E}_{i}^{2}-H^{i} C \mathcal{E}_{i}^{2}\right) \omega_{k}^{i, 2}-H^{i} F \eta_{k+1} \\
& -M^{i} K_{1}^{i} F \eta_{k}
\end{aligned}
$$

with $K^{i}=K_{1}^{i}+K_{2}^{i}$. Similar to (8), the parametric matrices for the $i$ th UIO can be obtained by solving

$$
\begin{aligned}
\mathcal{E}_{i}^{1}-H^{i} C \mathcal{E}_{i}^{1} & =\mathbf{0} \\
\mathcal{B}_{i}-M^{i} T^{i}-H^{i} C \mathcal{B}_{i} & =\mathbf{0} \\
\left(A-H^{i} C A-M^{i} K_{1}^{i} C\right) M^{i}-M^{i} N^{i} & =\mathbf{0} \\
\left(A-H^{i} C A-M^{i} K_{1}^{i} C\right) H^{i}-M^{i} K_{2}^{i} & =\mathbf{0} .
\end{aligned}
$$

Remark 6: The $i$ th UIO (10) is not affected by faults in the $i$ th actuator. Even if the $i$ th actuator has become faulty, the state estimation error will not be affected. Moreover, as pointed in Remark 3, the $i$ th UIO corresponds to not only the $i$ th actuator-fault situation but also to the healthy situation.

It is known that the UIO (10) can only decouple the effect of $\omega_{k}^{i, 1}$ from the residual signals, but cannot distinguish the effect of $\omega_{k}^{i, 2}$ from the faults on the residuals. This implies that if we only use the UIO (10), it is impossible to achieve FDI robustness with respect to $\omega_{k}^{i, 2}$. In order to implement robust FDI, we further propose to use the set-theoretic methods to passively consider the effect of $\omega_{k}^{i, 2}$ on the residual signals.

Assumption 7: The vector $\omega_{k}^{i, 2}$ is bounded by a known set $W^{i, 2}=\left\{\omega_{k}^{i, 2} \in \mathbb{R}^{n^{i}}:\left|\omega^{i, 2}-\omega^{i, 2, c}\right| \leq \bar{\omega}^{i, 2}\right\}$, where $\omega^{i, 2, c}$ and $\bar{\omega}^{i, 2}$ are known and constant vectors.

Based on (11) and (12), the dynamics of the state estimation error vector of the UIO (10) can be reduced into

$$
\begin{aligned}
e_{k+1}^{i}= & \left(A-H^{i} C A-M^{i} K_{1}^{i} C\right) e_{k}^{i}+\left(\mathcal{E}_{i}^{2}-H^{i} C \mathcal{E}_{i}^{2}\right) \omega_{k}^{i, 2} \\
& -H^{i} F \eta_{k+1}-M^{i} K_{1}^{i} F \eta_{k} .
\end{aligned}
$$


In the proposed FD method, the residual vector corresponding to the $i$ th UIO can be defined as

$$
r_{k}^{i}=y_{k}-\hat{y}_{k}^{i}=C e_{k}^{i}+F \eta_{k} .
$$

Since $\omega_{k}^{i, 2}, \eta_{k}$, and $\eta_{k+1}$ are bounded by $W^{i, 2}$ and $V$, a setbased version of (13) can be obtained as

$$
\begin{aligned}
E_{k+1}^{i}= & \left(A-H^{i} C A-M^{i} K_{1}^{i} C\right) E_{k}^{i} \oplus\left(\mathcal{E}_{i}^{2}-H^{i} C \mathcal{E}_{i}^{2}\right) W^{i, 2} \\
& \oplus\left(-H^{i} F V\right) \oplus\left(-M^{i} K_{1}^{i} F V\right),
\end{aligned}
$$

where $\oplus$ denotes the Minkowski sum and $E_{k}^{i}$ is the set of $e_{k}^{i}$ and $e_{k}^{0} \in E_{0}^{i}$ is given. With (15), a robust state estimation set $\hat{X}_{k}^{i}$ corresponding to the $i$ th SUIO can be obtained as $\hat{X}_{k}^{i}=$ $\left\{\hat{x}_{k}^{i}\right\} \oplus E_{k}^{i}$. Note that, according to [23], as $k$ tends to $\infty$, (15) converges to robust positively invariant (RPI) sets of (13). Thus, with the notion of RPI sets, we can construct an RPI set for $e_{k}^{i}$ based on (13), which is denoted as $E^{i}$ corresponding to the $i$ th SUIO. For the notion and computation of RPI sets, the readers are referred to [23] and [24].

Since when the system operates at steady state, $e_{k}^{i}$ is always inside the RPI set $E^{i}$, a steady-state set for $r_{k}^{i}$ can be further constructed using (14) as

$$
R^{i}=C E^{i} \oplus F V .
$$

\section{B. Fault Detection}

Definition 8 (SUIO): In the case that the system (1) cannot satisfy the existence conditions in Theorem 4, a UIO (10) able to decouple part of the unknown inputs of the system (1), handle the remaining unknown inputs using the set theory, and generate robust state estimation sets is named in this paper as the SUIO of the system (1), which is mathematically described by (10) and (13)-(16).

In the proposed FDI method, a bank of SUIOs are designed, each corresponding to not only faults in one actuator but also to the healthy situation. Particularly, faults in the $i$ th actuator is treated as an unknown input and the $i$ th SUIO is designed to be insensitive to the faults in the $i$ th actuator. Thus, $p$ SUIOs should be designed to monitor the faulty situations related to the $p$ actuators, respectively. During the system operation, actuator faults can be detected and isolated by combining the system-operating information from all the $p$ SUIOs. Since there are $p$ SUIOs, we should construct $p$ RPI sets, each describing the status of an actuator. Moreover, we can obtain $p$ residuals, each corresponding to one actuator as well.

Because $f_{i} u_{k}^{i}$ is considered as an unknown input decoupled by the $i$ th SUIO and the case that $f_{i}$ takes the value 1 corresponds to the healthy situation, all the SUIOs can also match the healthy actuator situation in addition to the faulty situations. Thus, when the system is at steady state of the healthy operation, we can have

$$
r_{k}^{i} \in R^{i} \text { for all } i \in \mathbb{I}=1,2, \ldots, p
$$

which means that if a violation of any inclusion out of the $p$ inclusions in (17) is detected, it can be guaranteed that the system has become faulty. Otherwise, it is assumed that the system is still healthy. Since all the SUIOs are used for FD in the proposed scheme, theoretically, its FDI sensitivity is higher when compared with some traditional observer-based schemes that only use the residual signal generated by the observer matching the current system situation for robust FDI.

\section{Fault Isolation}

In the previous section, when the system is healthy, the residual signals generated by all the SUIOs should be inside their corresponding RPI sets at steady state, respectively. Once a residual vector leaves its RPI set, it implies that the system has become faulty. However, since in the proposed method, each SUIO is designed to be insensitive to faults in one actuator, under the single-fault assumption, this means that the actuator corresponding to this residual signal leaving its RPI set must not become faulty. Thus, for making FI decisions, we should directly exclude this actuator.

With the logic explained above, we can remove all the excluded actuators from the set of candidate faulty actuators. Then, it can be guaranteed that the remaining actuators include the real faulty actuator, which is the first step of the proposed FI strategy. Afterwards, the set of actuators is divided into two groups (i.e., the healthy and fault-candidate actuators). The goal of the proposed FI method is to isolate the faulty actuator among the group of fault-candidate actuators.

When the system is healthy, for the $i$ th SUIO, the state estimation error of this SUIO has been derived as (13). Moreover, without faults, for any SUIO out of the $p$ SUIOs, its state estimation error is always subject to the same dynamics as in (13). If the $i$ th actuator becomes faulty, from the healthy situation to the $i$ th faulty situation, the state estimation error corresponding to the $i$ th SUIO will not be affected.

However, if the $j$ th actuator $(j \neq i)$ becomes faulty, the state estimation error of the $i$ th SUIO will change. The state equation of the plant under the $j$ th actuator fault is

$$
x_{k+1}=A x_{k}+\mathcal{B}_{j} u_{k}+\mathcal{E}_{j} \omega_{k}^{j}
$$

where $\mathcal{B}_{j}=B_{j} \mathcal{F}_{j}, \mathcal{E}_{j}=\left[b_{j} E\right]$ and $\omega_{k}^{j}=\left[f_{j} u_{k}^{j} \omega_{k}\right]^{T}$.

Furthermore, we can equivalently transform (18) into

$$
\begin{aligned}
x_{k+1} & =A x_{k}+\mathcal{B}_{j} u_{k}+\mathcal{E}_{j} \omega_{k}^{j} \\
& =A x_{k}+\mathcal{B}_{i} u_{k}+\mathcal{E}_{i} \omega_{k}^{i}+\delta_{k}^{j, i}
\end{aligned}
$$

with $\delta_{k}^{j, i}=\left(b_{j} f_{j}-b_{j}\right) u_{k}^{j}-\left(b_{i} f_{i}-b_{i}\right) u_{k}^{i}$.

With (13) and (19), when the $j$ th actuator becomes faulty instead of the $i$ th actuator, the dynamics of the state estimation error of the $i$ th SUIO can be derived as

$$
\begin{aligned}
e_{k+1}^{j, i}= & \left(A-H^{i} C A-M^{i} K_{1}^{i} C\right) e_{k}^{j, i} \\
& +\left(\mathcal{E}_{i}^{2}-H^{i} C \mathcal{E}_{i}^{2}\right) \omega_{k}^{i, 2}-M^{i} K_{1}^{i} F \eta_{k}-H^{i} F \eta_{k+1} \\
& +\left(I-H^{i} C\right) \delta_{k}^{j, i} .
\end{aligned}
$$

Thus, for the $i$ th SUIO, if we consider all the possible actuator faults except for the $i$ th one, the following dynamics can be obtained to describe the state estimation error under the different faulty situations:

$$
\zeta_{k+1}^{i}=G^{i} \zeta_{k}^{i}+Q^{i} u_{k}+S^{i} v^{i}
$$


where

$$
\begin{aligned}
& P^{i}=I-H^{i} C, \quad \bar{A}^{i}=A-H^{i} C A-M^{i} K_{1}^{i} C \\
& J_{l}=b_{l} f_{l}-b_{l}
\end{aligned}
$$

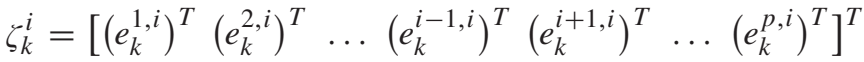

$$
\begin{aligned}
& G^{i}=\left[\begin{array}{ccccc}
\bar{A}^{i} & \mathbf{0} & \mathbf{0} & \ldots & \mathbf{0} \\
\mathbf{0} & \bar{A}^{i} & \mathbf{0} & \ldots & \mathbf{0} \\
\vdots & \vdots & \vdots & \ldots & \vdots \\
\mathbf{0} & \mathbf{0} & \mathbf{0} & \cdots & \bar{A}^{i}
\end{array}\right] \\
& S^{i}=\left[\begin{array}{ccc}
\left(\mathcal{E}_{i}^{2}-H^{i} C \mathcal{E}_{i}^{2}\right) & -M^{i} K_{1}^{i} F & -H^{i} F \\
\left(\mathcal{E}_{i}^{2}-H^{i} C \mathcal{E}_{i}^{2}\right) & -M^{i} K_{1}^{i} F & -H^{i} F \\
\vdots & \vdots & \vdots \\
\left(\mathcal{E}_{i}^{2}-H^{i} C \mathcal{E}_{i}^{2}\right) & -M^{i} K_{1}^{i} F & -H^{i} F
\end{array}\right] \\
& Q^{i}=\left[\begin{array}{ccccccccc}
P^{i} J_{1} & \mathbf{0} & \mathbf{0} & \cdots & \mathbf{0} & -P^{i} J_{i} & \mathbf{0} & \cdots & \mathbf{0} \\
\mathbf{0} & P^{i} J_{2} & \mathbf{0} & \cdots & \mathbf{0} & -P^{i} J_{i} & \mathbf{0} & \cdots & \mathbf{0} \\
\vdots & \vdots & \vdots & \cdots & \vdots & \vdots & \vdots & \cdots & \vdots \\
\mathbf{0} & \mathbf{0} & \mathbf{0} & \cdots & \mathbf{0} & -P^{i} J_{i} & \mathbf{0} & \cdots & P^{i} J_{p}
\end{array}\right] \\
& v^{i}=\left[\begin{array}{ll}
\left(\omega_{k}^{i, 2}\right)^{T} & \left.\left(\eta_{k}\right)^{T} \quad\left(\eta_{k+1}\right)^{T}\right]
\end{array}\right.
\end{aligned}
$$

Since $v^{i}$ is bounded, for given fault magnitudes $f_{1}, f_{2}, \ldots$, $f_{p}$, by designing $u_{k}$, an RPI set $\bar{E}^{i}$ for $\zeta_{k}^{i}$ can be constructed. Thus, if we can guarantee that

$$
\bar{R}^{i} \cap R^{i}=\varnothing
$$

where $\bar{R}^{i}$ is an union of $p-1$ sets of the residual vectors of the $i$ th SUIO, each set corresponding to a faulty situation in an actuator different from the $i$ th one. This implies that, for any considered fault except for the one in the $i$ th actuator, as long as it occurs, the residual vector of the $i$ th SUIO will leave its set $R^{i}$ and we can compute $\bar{R}^{i}$ as

$$
\bar{R}^{i}=\bar{R}^{1, i} \cup, \ldots, \cup \bar{R}^{i-1, i} \cup \bar{R}^{i+1, i} \cup, \ldots, \cup \bar{R}^{p, i}
$$

where $\bar{R}^{j, i}$ represents the set of the residual vector of the $i$ th SUIO in the $j$ th actuator-fault mode with

$$
\bar{R}^{j, i}=C \bar{E}^{j, i} \oplus F V
$$

where $\bar{E}^{j, i}$ is the RPI set of the state estimation error of the $i$ th SUIO in the $j$ th actuator-fault mode.

Remark 9: The considered fault magnitudes $f_{1}, f_{2}, \ldots, f_{p}$ are those critical to system performance/safety, which can be scalars or intervals (e.g., $f_{i}=0.5$ or $f_{i}=[0.1,0.5]$ ).

In order to satisfy (22), after FD, we need to design a proper control input vector for FI. In this paper, we design the control input for FI as a constant vector denoted as $u_{\text {const }}$ instead of a variable vector for simplicity. In this case, the RPI set $\bar{E}^{i}$ can have a smaller size and the selection of $u_{\text {const }}$ will only change the center of $\bar{E}^{i}$ not its size, which can reduce the conservatism of the guaranteed FI condition (22).

For each actuator, we have to guarantee that the corresponding condition (22) is satisfied. In this case, the FD and FI tasks can be guaranteed. A group of guaranteed FI conditions are summarized in Proposition 10.
Proposition 10: For the considered fault magnitudes $f_{1}, f_{2}$, $\ldots, f_{p}$, if there exists a constant input vector $u_{\text {const }}$ such that

$$
\bar{R}^{i} \cap R^{i}=\varnothing \text { for all } i=1,2, \ldots, p
$$

are satisfied, as long as one of the faults is detected, it is guaranteed that it can be isolated during a time interval by adjusting the system input into $u_{\text {const }}$ at the FD time instant.

Proof: If (25) is satisfied, after FD and injecting $u_{\text {const }}$ into the system, one and only one SUIO can generate residual signal that always stays in their corresponding RPI set, while the residual signals generated by the other SUIOs will leave their RPI sets, which guarantees the success of active FI.

The proposed FI strategy consists in changing the input vector to $u_{\text {const }}$ at the FD time instant and then testing which residual vector among the remaining group of SUIOs can always satisfy the inclusion (17). Finally, the residual vector can accurately indicate the fault by means of this testing.

Additionally, we should know that $u_{\text {const }}$ needs not only to satisfy the FI conditions in Proposition 10 but also some other constraints related to system safety/performance. In this paper, we use an input set $\mathscr{U}$ to generally describe the input constraints. Moreover, for the $i$ th SUIO, we further define the set of input vectors that can guarantee the satisfaction of (22) as $\mathbb{U}_{i}$, which means that if any input vector belonging to $\mathbb{U}_{i}$ is injected into the system after FD, allowing to know whether the $i$ th actuator has become faulty or not by testing (17). Thus, in order to distinguish faults in all the actuators, an input vector ensuring Proposition 10 must satisfy

$$
u_{\text {const }} \in \mathscr{U}=\bigcap_{i=1}^{p} \mathbb{U}_{i}
$$

where each input set $\mathbb{U}_{i}(i \neq 0)$ corresponds to an SUIO.

Although any $u_{\text {const }} \in \mathscr{U}$ can assure active FI, in order to reduce damages to the system during active FI as much as possible, we formulate the problem of selecting an optimal FI-oriented input vector $u_{\text {const }}^{*}$ as an optimization problem

$$
u_{\text {const }}^{*}=\inf \left\{u: u^{T} Q u, u \in \mathscr{U}\right\}
$$

where $Q$ is a positive semi-definite matrix and $u_{\text {const }}^{*}$ is based on the minimization of the energy of the designed input vector. A systematic solution of the optimization problem (27) will be developed as further research. However, a method designing an input vector for the problem (27) can be found in [25].

Finally, in order to help the readers understand the proposed FDI approach, the FDI procedure is formalized in Algorithm 1.

\section{Illustrative EXAMPLE}

We use a quadrotor manufactured by Draganfly Innovations to illustrate the proposed FDI method. The nonlinear model and its parameters are introduced, defined, and identified in [26], where the variables $\dot{x}, \dot{y}, \dot{z}, \phi, \theta, \psi, p, q, r$ are chosen as the states of the quadrotor and $\omega_{1}, \omega_{2}, \omega_{3}$ and $\omega_{4}$ are defined as the inputs of the quadrotor. Without loss of generality, we only consider the hovering status of the quadrotor here. At hovering, we set $\dot{x}=0, \dot{y}=0, \dot{z}=0$, $\phi=0, \theta=0, \psi=0, p=0, q=0, r=0$, and $U_{1}=m g(m$ 


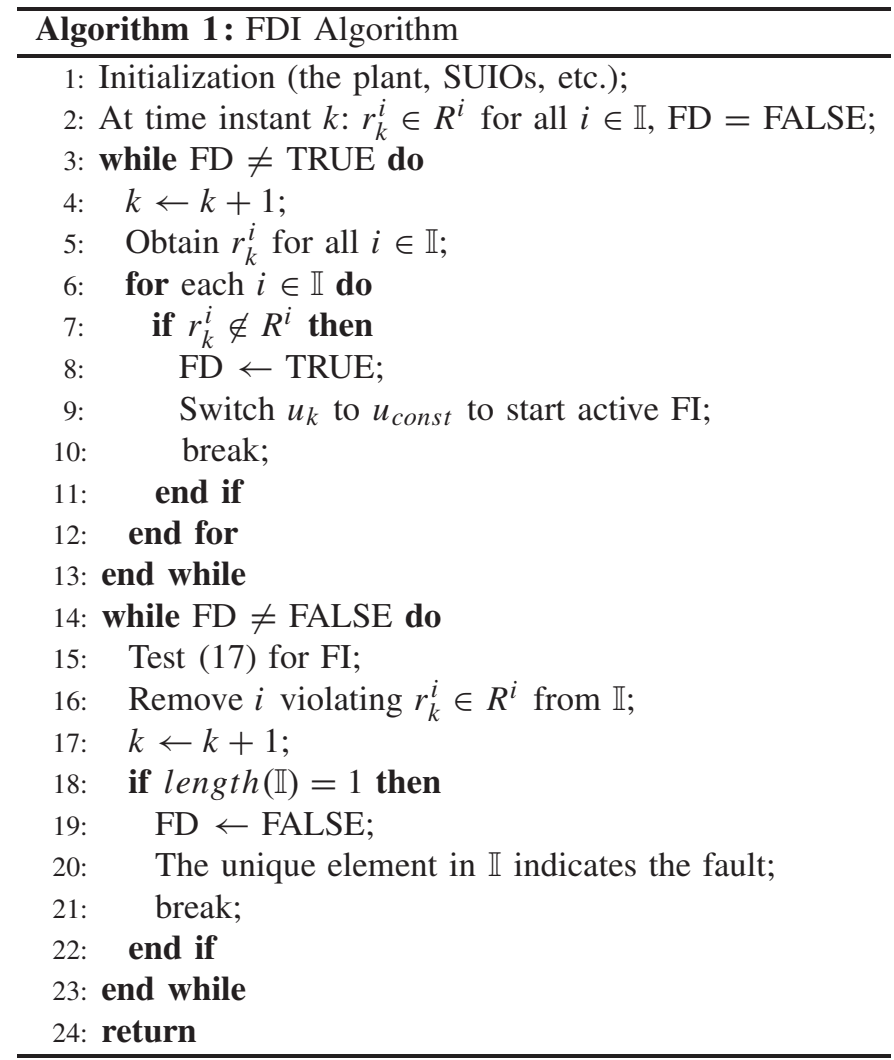

and $g$ are the mass and the gravity accelerator, respectively) and $\omega_{1}=\omega_{2}=\omega_{3}=\omega_{4}=181.2171 \mathrm{rad} / \mathrm{s}$. Consequently, we can linearize the nonlinear model around the hovering point obtaining a linear model. Furthermore, discretizing this linear model with a sampling time of $T=0.01 \mathrm{~s}$, we can obtain a linear time-invariant discrete model. For simplicity, we only use the subsystem describing the rotation around the body frame of the quadrotor as the case study and the outputs of the subsystem are chosen as $p, q$, and $r$. The model parameters in (1) for this subsystem are obtained as

$$
\begin{aligned}
A & =\left[\begin{array}{ccc}
0.9985 & 0 & 0 \\
0 & 0.9985 & 0 \\
0 & 0 & 0.9991
\end{array}\right], \quad C=\left[\begin{array}{lll}
1 & 0 & 0 \\
0 & 1 & 0 \\
0 & 0 & 1
\end{array}\right] \\
F & =\left[\begin{array}{lll}
1 & 0 & 0 \\
0 & 1 & 0 \\
0 & 0 & 1
\end{array}\right] \\
B & =\left[\begin{array}{cccc}
0 & 0.0058 & 0 & -0.0058 \\
-0.0058 & 0 & 0.0058 & 0 \\
-0.0002 & 0.0002 & -0.0002 & 0.0002
\end{array}\right] \\
E & =\left[\begin{array}{cccc}
0.5 & 0.3 & 0 & 1.7 \\
0.4 & 0 & 0.6 & 0 \\
0.3 & 0.2 & 0.05 & 0.06
\end{array}\right] .
\end{aligned}
$$

Since there are four actuators in the quadrotor, we consider four fault magnitude intervals corresponding to them, respectively, which are given as $f_{1}=[0.1,0.15], f_{2}=[0.2,0.25]$, $f_{3}=[0.1,0.15]$, and $f_{4}=[0.2,0.25]$. In this example, we use random fault magnitudes in real time inside the corresponding intervals given above. According to (3), the four considered
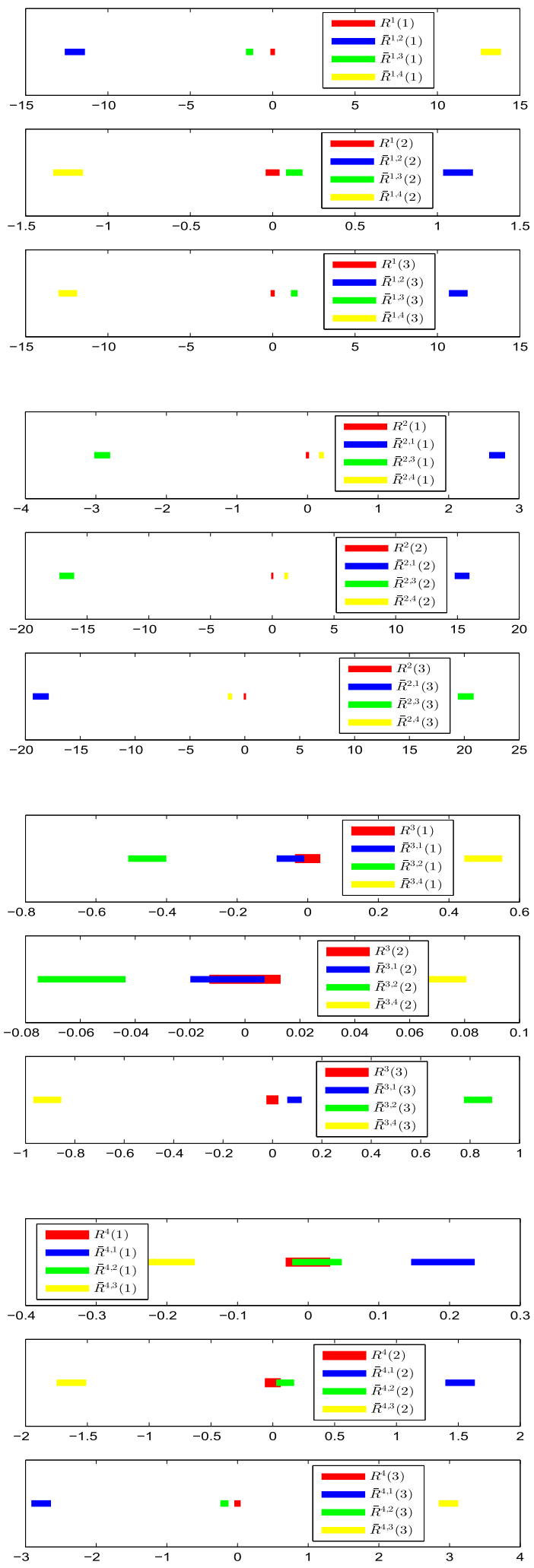

Fig. 1. Verification of the guaranteed FI conditions.

actuator modes are modeled as

$$
\mathcal{B}_{1}=\left[\begin{array}{cccc}
0 & 0.0058 & 0 & -0.0058 \\
0 & 0 & 0.0058 & 0 \\
0 & 0.0002 & -0.0002 & 0.0002
\end{array}\right]
$$




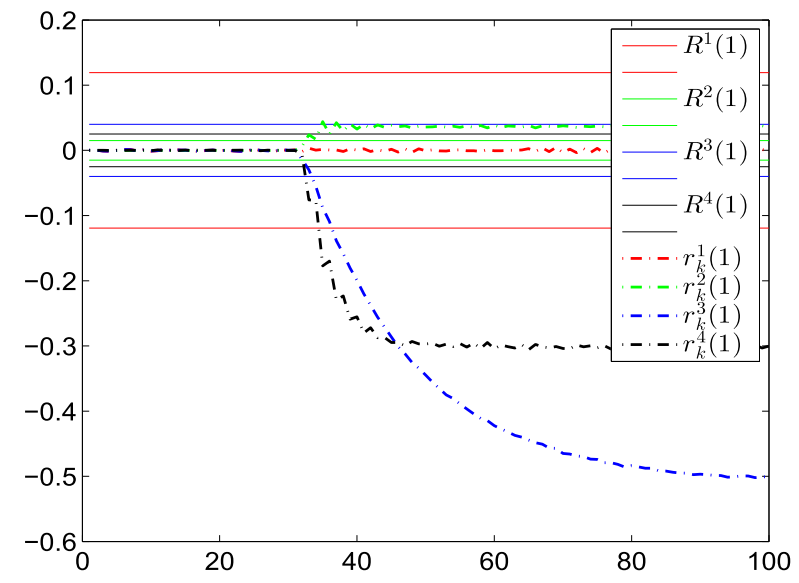

(a)

Fig. 2. Evaluation of the first component of the residuals of the four SUIOs (superindex indicates $\mathrm{SUIO} /$ the faulty mode).

$$
\begin{aligned}
& \mathcal{E}_{1}^{1}=\left[\begin{array}{cc}
0 & 0.5 \\
-0.0058 & 0.4 \\
-0.0002 & 0.3
\end{array}\right] \\
& \mathcal{B}_{2}=\left[\begin{array}{cccc}
0 & 0 & 0 & -0.0058 \\
-0.0058 & 0 & 0.0058 & 0 \\
-0.0002 & 0 & -0.0002 & 0.0002
\end{array}\right] \\
& \mathcal{E}_{2}^{1}=\left[\begin{array}{cc}
0.0058 & 0.5 \\
0 & 0.4 \\
0.0002 & 0.3
\end{array}\right] \\
& \mathcal{B}_{3}=\left[\begin{array}{cccc}
0 & 0.0058 & 0 & -0.0058 \\
-0.0058 & 0 & 0 & 0 \\
-0.0002 & 0.0002 & 0 & 0.0002
\end{array}\right] \\
& \mathcal{E}_{3}^{1}=\left[\begin{array}{cc}
0 & 0.5 \\
0.0058 & 0.4 \\
-0.0002 & 0.3
\end{array}\right] \\
& \mathcal{B}_{4}=\left[\begin{array}{cccc}
0 & 0.0058 & 0 & 0 \\
-0.0058 & 0 & 0.0058 & 0 \\
-0.0002 & 0.0002 & -0.0002 & 0
\end{array}\right] \\
& \mathcal{E}_{4}^{1}=\left[\begin{array}{cc}
-0.0058 & 0.5 \\
0 & 0.4 \\
0.0002 & 0.3
\end{array}\right] \\
& \mathcal{E}_{1}^{2}=\mathcal{E}_{2}^{2}=\mathcal{E}_{3}^{2}=\mathcal{E}_{4}^{2}=\left[\begin{array}{ccc}
0.3 & 0 & 1.7 \\
0 & 0.6 & 0 \\
0.2 & 0.0 .5 & 0.06
\end{array}\right] \text {. }
\end{aligned}
$$

The effect of disturbances, noises, and linearizing errors are considered to be bounded in the sets of $\eta_{k}$ and $\omega_{k}^{i, 2}(i=1$, 2,3 , and 4) are given as $V=\left\{\eta:-\left[\begin{array}{lll}2 & 2 & 2\end{array}\right]^{T} \times 10^{-3} \leq \eta \leq\right.$ $\left.\left[\begin{array}{lll}2 & 2 & 2\end{array}\right]^{T} \times 10^{-3}\right\}$ and $W^{i, 2}=\left\{\omega^{i, 2}:-\left[\begin{array}{lll}2 & 2 & 2\end{array}\right]^{T} \times 10^{-3} \leq\right.$ $\left.\omega^{i, 2} \leq\left[\begin{array}{lll}2 & 2 & 2\end{array}\right]^{T} \times 10^{-3}\right\}$, respectively.

Four SUIOs are designed to monitor four motors, each corresponding to one considered fault interval (i.e., $f_{1}, f_{2}$, $f_{3}$, and $f_{4}$ ). If we consider a fault magnitude interval for each actuator, it means that as long as the proposed FI conditions are satisfied, any actuator fault magnitude in the interval can be isolated by the proposed FI approach. The parameters of

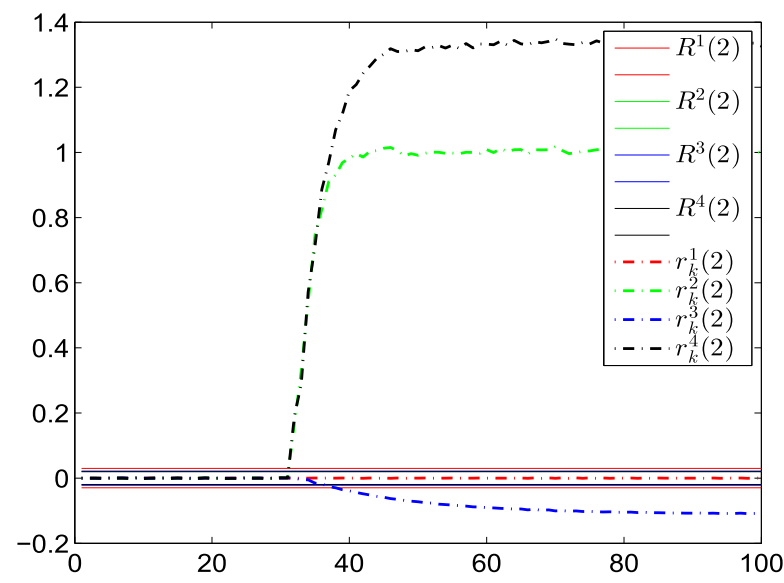

(a)

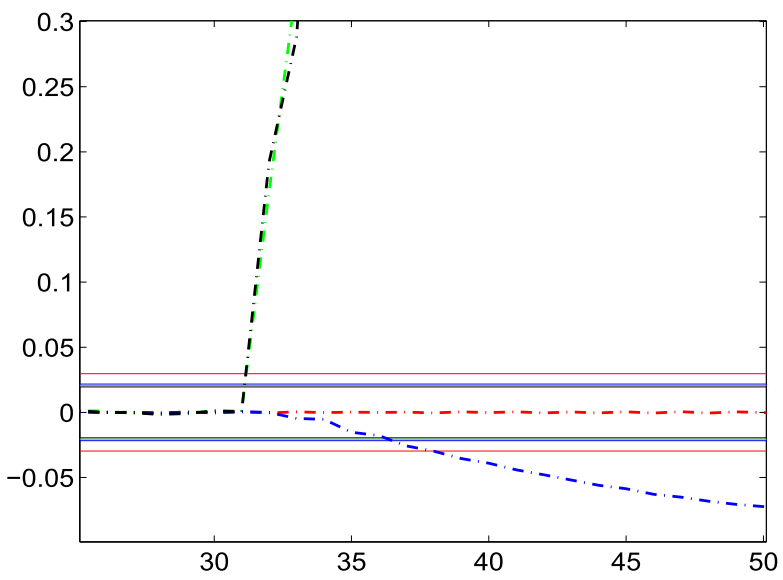

(b)

Fig. 3. Evaluation of the second component of the residuals of the four SUIOs (superindex indicates SUIO/the faulty mode). (a) Second component of residual. (b) Detail of the second residual component.

the four SUIOs are designed as

$$
\begin{aligned}
& N^{1}=\left[\begin{array}{ccc}
5.7257 & 6.7741 & 5.7676 \\
-4.7021 & -5.6668 & -5.4171 \\
-0.6817 & -0.6970 & 0.3279
\end{array}\right] \\
& H^{1}=\left[\begin{array}{ccc}
0.7502 & -0.0123 & 0.4327 \\
-0.0123 & 0.9994 & 0.0213 \\
0.4327 & 0.0213 & 0.2504
\end{array}\right] \\
& T^{1}=\left[\begin{array}{cccc}
0 & 0.0594 & 0.0061 & -0.0655 \\
0 & -0.0735 & -0.0076 & 0.0811 \\
0 & 0.0339 & 0.0035 & -0.0374
\end{array}\right] \\
& M^{1}=\left[\begin{array}{ccc}
0.7050 & 0.809 & 0.56 \\
0.09 & 0.27 & 0.43 \\
0.58 & 0.69 & 0.41
\end{array}\right] \\
& K_{1}^{1}=\left[\begin{array}{ccc}
-1.9069 & -2.0134 & 0.2479 \\
1.4133 & 3.7500 & -0.3910 \\
0.5232 & -1.8918 & 0.9533
\end{array}\right] \\
& K_{2}^{1}=\left[\begin{array}{ccc}
1.2940 & 1.9833 & 0.8033 \\
-0.8394 & -3.7218 & -0.5901 \\
-0.8308 & 1.8767 & -0.4264
\end{array}\right]
\end{aligned}
$$




$$
\begin{aligned}
& N^{2}=\left[\begin{array}{ccc}
-0.9789 & -1.2320 & -1.8896 \\
1.1277 & 1.1467 & 1.2675 \\
-1.0319 & -0.8549 & -0.3831
\end{array}\right] \\
& H^{2}=\left[\begin{array}{ccc}
0.9995 & -0.0134 & 0.0188 \\
-0.0134 & 0.6622 & 0.4728 \\
0.0188 & 0.4728 & 0.3384
\end{array}\right] \\
& T^{2}=\left[\begin{array}{cccc}
-0.0075 & 0 & 0.0081 & -0.0006 \\
0.0005 & 0 & -0.0006 & 0 \\
0.0069 & 0 & -0.0075 & 0.0006
\end{array}\right] \\
& M^{2}=\left[\begin{array}{ccc}
0.6 & 0.8 & 0.58 \\
0.5 & 0.27 & 0.25 \\
0.4 & 0.19 & 0.8
\end{array}\right] \\
& K_{1}^{2}=\left[\begin{array}{ccc}
0.2296 & -0.0856 & 0.0104 \\
0.1005 & -0.2476 & -0.1269 \\
0.1154 & 0.5933 & 0.5668
\end{array}\right] \\
& K_{2}^{2}=\left[\begin{array}{ccc}
-0.2309 & 0.0544 & 0.0323 \\
-0.1014 & 0.2253 & 0.1581 \\
-0.1180 & -0.6588 & -0.47416
\end{array}\right] \\
& N^{3}=\left[\begin{array}{ccc}
-54.214 & -52.1073 & -92.2285 \\
145.531 & 139.0987 & 245.3171 \\
-51.654 & -48.7388 & -85.2332
\end{array}\right] \\
& H^{3}=\left[\begin{array}{ccc}
0.7208 & 0.0127 & 0.4484 \\
0.0127 & 0.9994 & -0.0204 \\
0.4484 & -0.0204 & 0.2798
\end{array}\right] \\
& T^{3}=\left[\begin{array}{cccc}
-0.0099 & -0.1040 & 0 & 0.1140 \\
0.0259 & 0.2714 & 0 & -0.2973 \\
-0.0087 & -0.0911 & 0 & 0.0998
\end{array}\right] \\
& M^{3}=\left[\begin{array}{lll}
0.5 & 0.6 & 1.2 \\
1.3 & 0.8 & 0.9 \\
1.5 & 0.7 & 0.4
\end{array}\right] \\
& K_{1}^{3}=\left[\begin{array}{ccc}
41.909 & 24.371 & 25.74 \\
-111.884 & -65.187 & -67.648 \\
38.995 & 22.98 & 23.135
\end{array}\right] \\
& K_{2}^{3}=\left[\begin{array}{ccc}
-42.052 & -24.364 & -25.494 \\
111.787 & 65.191 & 67.759 \\
-38.766 & -22.994 & -23.487
\end{array}\right] \\
& N^{4}=\left[\begin{array}{ccc}
0.9253 & 0.3544 & 0.8586 \\
-0.7457 & -0.3121 & -0.8786 \\
-0.1374 & -0.5212 & -0.6305
\end{array}\right] \\
& H^{4}=\left[\begin{array}{ccc}
0.9995 & 0.0138 & -0.0175 \\
0.0138 & 0.6187 & 0.4855 \\
-0.0175 & 0.4855 & 0.3818
\end{array}\right] \\
& T^{4}=\left[\begin{array}{cccc}
0.0108 & 0.0008 & -0.0116 & 0 \\
-0.0065 & -0.0005 & 0.007 & 0 \\
0.0003 & 0 & -0.0003 & 0
\end{array}\right] \\
& M^{4}=\left[\begin{array}{ccc}
0.3 & 0.5 & 0.4 \\
0.12 & 0.56 & 0.89 \\
0.45 & 0.36 & 0.8
\end{array}\right] \\
& K_{1}^{4}=\left[\begin{array}{ccc}
-0.3668 & -0.8526 & 0.4048 \\
0.0207 & 0.5184 & 0.3019 \\
0.4184 & 0.6066 & -0.0876
\end{array}\right] \\
& K_{2}^{4}=\left[\begin{array}{ccc}
0.3855 & 0.3366 & 0.2535 \\
-0.0225 & -0.4680 & -0.3669 \\
-0.4280 & -0.3384 & -0.2537
\end{array}\right] \text {. }
\end{aligned}
$$

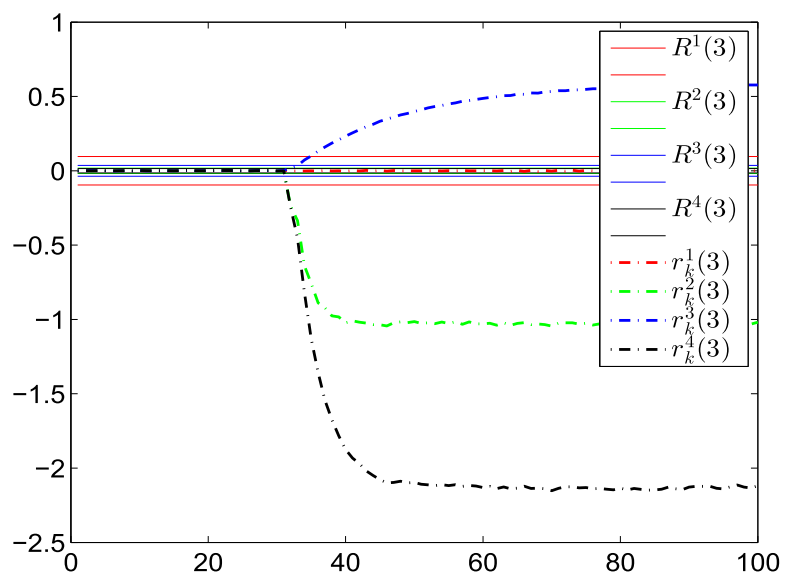

(a)

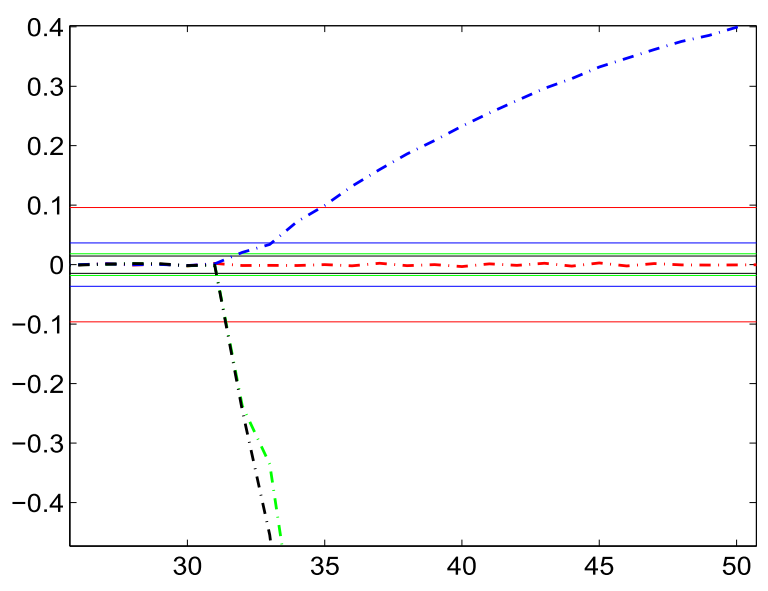

(b)

Fig. 4. Evaluation of the third component of the residuals of the four SUIOs (superindex indicates SUIO/the faulty mode). (a) Third component of residual. (b) Detail of the third residual component.

Based on the FI conditions in Proposition 10, a constant input vector $u_{\text {const }}=(200,200,200,200)^{T}$ is designed for active FI. Using $u_{\text {const }}$, the residual sets of the SUIOs can be constructed as in Fig. 1. Notice that, in Fig. 1, the first three subplots, the second three subplots, the third three subplots, and the fourth three subplots correspond to the first SUIO, the second SUIO, the third SUIO, and the fourth SUIO, respectively. Taking the first SUIO as an example, we can see that $R^{1} \cap \bar{R}^{1,2}=\varnothing, R^{1} \cap \bar{R}^{1,3}=\varnothing$, and $R^{1} \cap \bar{R}^{1,4}=\varnothing$, which implies $R^{1} \cap \bar{R}^{1}=\varnothing$. Furthermore, in the remaining subplots, we can observe that $R^{2} \cap \bar{R}^{2}=\varnothing, R^{3} \cap \bar{R}^{3}=\varnothing$, and $R^{4} \cap \bar{R}^{4}=\varnothing$. Thus, $u_{\text {const }}$ can satisfy the FI conditions. Note that each subplot in Fig. 1 describes one component of the corresponding sets, and the notations $R^{i}(l)$ and $\bar{R}^{i, j}(l)$ denote the $l$ th component intervals of $R^{i}$ and $\bar{R}^{i, j}$, respectively. The meaning of $R^{i}$ and $\bar{R}^{i, j}$ can be understood according to (22) and (23). Since all the intervals are 1-D, only the horizonal axis of coordinate frame is used.

In this paper, we consider a fault scenario that, from $k=1$ to $k=30$, the system is healthy, but at $k=31$, the first actuator becomes faulty. The real-time fault magnitude set 
in the simulation is $f_{1}=0.1+0.05 \mathrm{rand}$, where rand is a random value inside the interval $[0,1]$. The simulation results corresponding to this fault scenario are shown in Figs. 2-4. It can be observed that a fault is detected at time instant $k=32$ (i.e., $r_{32}^{1}(2) \in R^{1}(2), r_{32}^{2}(2) \notin R^{2}(2), r_{32}^{3}(2) \in R^{3}(2)$, and $r_{32}^{4}(2) \notin R^{4}(2)$ ). Thus, at time instant $k=32$ (i.e., the FD time), we directly adjust the system input to $u_{\text {const }}$ (i.e., enter into the phase of active FI). Furthermore, it can be concluded that the first actuator has become faulty at $k=34$ based on the proposed active FI strategy in Fig. 4 (i.e., $r_{34}^{1}(3) \in R^{1}(3)$, $r_{34}^{2}(3) \notin R^{2}(3), r_{34}^{3}(3) \notin R^{3}(3)$, and $r_{34}^{4}(3) \notin R^{4}(3)$, where only the residual of the first SUIO is still inside its set while the residuals generated by the other three SUIOs have gone out of their sets), which show the effectiveness of the proposed FDI method.

\section{CONCLUSION}

In this paper, a new robust FDI method combining UIOs and invariant sets is proposed to detect and isolate actuator faults based on a bank of SUIOs, where we consider faults and disturbances as unknown inputs and divide them into two groups. The first group includes unknown inputs that can be actively decoupled by UIOs. For the second group, we use their bounds to passively propagate their effect to the residual sets. The advantage of the proposed method is that all the SUIOs are used for FD. Moreover, it should be more sensitive to faults than some existing set-theoretic methods that only use the observer matching the current system situation. For FI, the proposed method designs an input vector and uses it to actively force the residual vectors of SUIOs to leave their sets if they do not match the faulty mode, which can guarantee FI and reduce FI conservatism. In the future, we will consider multiple faults and implement SUIO-based faulttolerant control.

\section{REFERENCES}

[1] M. Blanke, M. Kinnaert, J. Lunze, and M. Staroswiecki, Diagnosis and Fault-Tolerant Control. Berlin, Germany: Springer-Verlag, 2006.

[2] B. Xiao and S. Yin, "An intelligent actuator fault reconstruction scheme for robotic manipulators," IEEE Trans. Cybern., to be published.

[3] J. Chen, R. J. Patton, and H.-Y. Zhang, "Design of unknown input observers and robust fault detection filters," Int. J. Control, vol. 63, no. 1 , pp. 85-105, 1996.

[4] V. Puig, "Fault diagnosis and fault tolerant control using set-membership approaches: Application to real case studies," Int. J. Appl. Math. Comput. Sci., vol. 20, no. 4, pp. 619-635, 2010.

[5] R. Seydou, T. Raïsi, A. Zolghadri, D. Efimov, and C. Combastel, "Robust fault diagnosis based on constraint satisfaction and interval continuoustime parity equations," in Proc. 8th IFAC Symp. Fault Detection, Supervision Safety Tech. Process., Aug. 2012, pp. 1293-1298.

[6] F. Xu, V. Puig, C. Ocampo-Martinez, F. Stoican, and S. Olaru, "Closedloop actuator-fault detection and isolation using invariant sets and tubes," in Proc. 19th IFAC World Congr., Cape Town, South Africa, Aug. 2014, pp. $8020-8035$.
[7] S. Hui and S. Żak, "Observer design for systems with unknown inputs," Int. J. Appl. Math. Comput. Sci., vol. 15, no. 4, pp. 431-446, 2005.

[8] Z. Gao, X. Liu, and M. Z. Q. Chen, "Unknown input observer-based robust fault estimation for systems corrupted by partially decoupled disturbances," IEEE Trans. Ind. Electron., vol. 63, no. 4, pp. 2537-2547, Apr. 2015.

[9] H. Behzad, A. Casavola, F. Tedesco, and M. A. Sadrnia, "A fault-tolerant sensor reconciliation scheme based on LPV unknown input observers," in Proc. 42nd IEEE Conf. Decision Control, Las Vegas, NV, USA, Dec. 2016, pp. 2158-2163.

[10] P. Kudva, N. Viswanadham, and A. Ramakrishna, "Observers for linear systems with unknown inputs," IEEE Trans. Autom. Control, vol. AC-25, no. 1, pp. 113-115, Feb. 1980.

[11] B. A. Charandabi and H. J. Marquez, "A novel approach to unknown input filter design for discrete-time linear systems," Automatica, vol. 50, no. 11 , pp. 2835-2839, 2014

[12] T.-G. Park, "Designing fault detection observers for linear systems with mismatched unknown inputs," J. Process Control, vol. 23, no. 8, pp. 1185-1196, 2013.

[13] D. Ichalal and S. Mammar, "On unknown input observers for LPV systems," IEEE Trans. Ind. Electron., vol. 62, no. 9, pp. 5870-5880, Sep. 2015.

[14] C. Ocampo-Martinez, J. A. De Doná, and M. M. Seron, "Actuator faulttolerant control based on set separation," Int. J. Adaptive Control Signal Process., vol. 24, no. 12, pp. 1070-1090, 2010.

[15] M. M. Seron, J. A. De Doná, and S. Olaru, "Fault tolerant control allowing sensor healthy-to-faulty and faulty-to-healthy transitions," IEEE Trans. Autom. Control, vol. 57, no. 7, pp. 1657-1669, Jul. 2012

[16] F. Stoican, M. M. S. Seron Olaru, and J. A. De Doná, "Reference governor design for tracking problems with fault detection guarantees," J. Process Control, vol. 22, no. 5, pp. 829-836, 2012.

[17] V. Puig, J. Quevedo, T. Escobet, and A. Stancu, "Robust fault detection using linear interval observers," in Proc. IFAC SafeProcess, Washington, DC, USA, Jun. 2003, pp. 609-614.

[18] V. Puig, A. Stancu, and J. Quevedo, "Observers for interval systems using set and trajectory-based approaches," in Proc. 44th IEEE Conf. Decision Control Eur. Control Conf. (CDC-ECC), Dec. 2005, pp. 6567-6572.

[19] J. Blesa, V. Puig, J. Romera, and J. Saludes, "Fault diagnosis of wind turbines using a set-membership approach," in Proc. 18th IFAC World Congr., Milano, Italy, 2011, pp. 8316-8321.

[20] P. Rosa and C. Silvestre, "Fault detection and isolation of LPV systems using set-valued observers: An application to a fixed-wing aircraft," Control Eng. Pract., vol. 21, no. 3, pp. 242-252, 2013.

[21] C. Combastel, V. Puig, and T. Raïssi, and T. Alamo, "Set-membership methods applied to FDI and FTC," Int. J. Adaptive Control Signal Process., vol. 30, no. 2, pp. 150-153, 2016.

[22] F. Xu, J. Tan, X. Wang, V. Puig, B. Liang, and B. Yuan, "A novel design of unknown input observers using set-theoretic methods for robust fault detection," in Proc. Amer. Control Conf., Boston, MA, USA, Jul. 2016, pp. 5957-5961.

[23] S. Olaru, J. A. De Doná, M. M. Seron, and F. Stoican, "Positive invariant sets for fault tolerant multisensor control schemes," Int. J. Control, vol. 83 , no. 12 , pp. 2622-2640, 2010.

[24] E. Kofman, H. Haimovich, and M. M. Seron, "A systematic method to obtain ultimate bounds for perturbed systems," Int. J. Control, vol. 80, no. 2, pp. 167-178, 2007 .

[25] K. J. Scott, R. Findeisen, D. R. Braatz, and M. D. Raimondo, "Input design for guaranteed fault diagnosis using zonotopes," Automatica, vol. 50, no. 6, pp. 1580-1589, 2014.

[26] L. Derafa, T. Madani, and A. Benallegue, "Dynamic modelling and experimental identification of four rotors helicopter parameters," in Proc. IEEE Int. Conf. Ind. Technol., Dec. 2006, pp. 1834-1839. 